\title{
Medical faculty members at different professorial levels
}

Student

How can we recognise certain distinguishing features of professors at different levels?

In academic medicine, the process of professorial promotion has previously proceeded in a predictable pattern. Senior faculty members have now become alarmed by the thought that a competency based approach might be applied to the world of academic medicine. ${ }^{12}$ The competencies and "metacompetencies" of senior faculty are complex and reflect such higher order thought processes that they cannot be meaningfully assessed by the demeaning use of superficial checklists. Given that a reductionist might be useful to depict the general characteristics and competencies of medical faculty at different professorial ranks. While this snapshot relies on generalities and stereotypes, it at least allows an observer who is unfamiliar with the system to recognise certain distinguishing features of faculty at different levels.

Competing interests: None declared.

1 Leung WC. Competency based medical training: review. BMJ 2002;325 693-5.

2 Diwakar V. Commentary: The baby is thrown out with the bathwater. BMJ 2002;325:695-6.

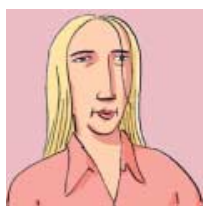

Assistant professo

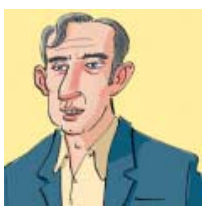

Associate professor

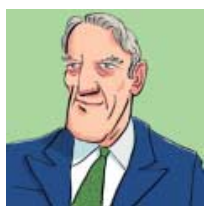

Full professo

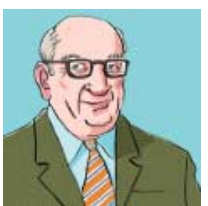

Emeritus professor

\section{General features}

Age (years)

Age (\%ears)

Spouse's occupation

$35^{\star}$

Spouse's name (female)

Spouse's name (male)

Annual salarył

Medication

Hair colour

Frequent flyer level

Mobile phone

Second language

45

45

linical competence

Knowledge test

Knowledge test

Modus operandi

Preferred method of diagnosis

Months of clinical service per year

Britney/Heather

John

John

$55-65$

55

The "olive" (of pyloric stenosis)

No medication

Teaching

Teaching style

Teaching award

Teaching interest

Delivers feedback to

Gold

Silver

Picture, email, etc

Spanish (or English)

Doctor or lawyer

65

Ashley/Jennifer

Housewife

75

Susan/Janet

John

$13 \mathrm{X}-1.5 \mathrm{X} \quad 0$ to $0.5 \mathrm{X}$

White pill (statin)

Purple pill (esomeprazole)

John

0 to $0.5 X$

Platinum Silver

Gold Platinum

1 per family

pill (sildenafil)

Not applicable (alopecia)

French or German

Latin

Varied

Difficult without amplification

\section{Research}

Favourite grant $\quad$ R-01 (NIH)

Grant stage

No of publications

No of authors per publication

Spell apoptosis

Pronounce apoptosis

Understand apoptosis

3

Authorship in $>50 \%$ of publications

Favourite lab

Understand proteomics

Define proteomics

Subspecialty referral

12

Open surgery

Laboratory testing

Consults paediatric surgeon

6

Orders ultrasound

Spell protein Spill protein

Alternatives to surgery Autopsy

Physical examination History

$10-12$

$\begin{array}{ll}1 & 0-12 \\ \text { Palpates with confidence } & \text { Diagnoses by history; orders Martini }\end{array}$

Favourite means of determining Logistic regression using SPSS

significance

Research meetings and reputation

10-minute preceptor

5-minute preceptor

1-minute preceptor

30-minute preceptor

Asks to teach

No

Loves to teach

Resident

No

Likes to teach

Yes

Faculty Dean

Hugh (or Amy) Cary Ulysses S

Gets renewed $\quad$ Reviews others $\quad$ Not applicable

Gets funded

dministrative features

Favourite dean

Secretarial support

Computer

Office

Organised medicine/medical society

25

6

Middle author

Last author Single author

Dry Clinic $\quad$ Black or chocolate

Non-parametric tests (such as rank $\chi^{2}$ test, $t$ test, or biostatistician tests, Fisher exact test)

Regional National
International or Rotary Club

${ }^{*}$ Age at first faculty appointment varies according to clinical specialty (for example, for neurosurgery, correction factor is +6 years). $†$ Age at retirement and transition to emeritus status varies inversely with both the night call requirements and the salary of the particular specialty. ‡Salary varies inversely with the prestige of the hiring university; regional differences may also apply. 